\title{
BRIGHT X-RAY STARS NEAR THE GALACTIC CENTER
}

\author{
MASAAKI SAKANO, MAMIKO NISHIUCHI, YOSHITOMO MAEDA, \\ KATSUJI KOYAMA AND JUN YOKOGAWA \\ Department of Physics, Kyoto University, Sakyo, Kyoto, Japan
}

\begin{abstract}
.
This paper report the $A S C A$ observations of the three brightest persistent X-ray stars near the Galactic Center: an X-ray burster A1742-294, black hole candidate 1E 1740.7-2942, and unclassified source 1E 1743.1-2843. Emission mechanism is briefly discussed based on the new ASCA results.
\end{abstract}

\section{Introduction}

The Galactic Center region draws much attention in every wave band, in conjunction with a possible massive black hole, starburst activity and so on. In order to overview the high energy phenomena, we have performed repeated $A S C A$ observations near the Galactic Center, consisting of a few tens of pointings with total exposure of about $1000 \mathrm{ksec}$. Several new facts are already published (e.g. Koyama et al. 1996). This paper is focused on the persistent X-ray stars locating near the Galactic Center. Systematic works on the whole X-ray stars in the Galactic Center region are presented by Sakano et al. (1997).

\section{Results \& Discussion}

\section{X-RAY BURSTER: A1742-294}

A1742-294 is the brightest persistent X-ray binary near the Galactic Center. Several X-ray bursts have been reported by Pavlinsky et al. (1994). We also detected 10 bursts in the total observation time of $115 \mathrm{ksec}$, hence confirmed A1742-294 to be an X-ray burster. All these bursts showed black body spectra with spectral softening in the decay phase, hence are established to be type-I bursts. At the Galactic Center distance $(8.5 \mathrm{kpc})$, the 
peak intensity of all the bursts is estimated to be below the Eddington limit of a neutron star. A1742-294 also exhibited a long-term variability by a factor of 3 in 3.5 years.

\section{E 1740.7-2942 ("THE GREAT ANNIHILATOR")}

$1 \mathrm{E}$ 1740.7-2942 is one of the most interesting sources in this region. It exhibits double radio jets (Mirabel et al., 1992), strong hard X-ray emission extending up to $100 \mathrm{keV}$ and intermittent $511 \mathrm{keV}$ bursts (Bouchet et al., 1991). The X-ray position coincides to the peak of a molecular cloud (Bally \& Leventhal, 1991). All together, 1E 1740.7-2942 is referred to be a micro quasar, powered by direct gas accretion from the molecular cloud.

We detected long-term flux variability by a factor of 3 , with no spectral change. The spectrum is found to be highly absorbed $\left(N_{H} \sim 1.5 \times\right.$ $10^{23} \mathrm{H} \mathrm{cm}^{-2}$ ), although the overall spectral shape with $1-10 \mathrm{keV}$ is not sufficiently well represented with a single-power law. No significant fluorescent line from neutral iron is found, suggesting that $1 \mathrm{E} 1740.7-2942$ is not in the dense cloud but would be behind it. Thus we infer that $1 \mathrm{E}$ 1740.7-2942 is a binary black hole rather than an isolated black hole in the dense cloud.

\section{UNCLASSIFIED OBJECT: 1E 1743.1-2843}

1E 1743.1-2843 has been observed many times in the past, but none is clear for the nature of this source. Although long-term variability was found with $A S C A$, neither short-term nor periodic variation was found. The spectrum is fitted by an absorbed power-law model with a photon index $\Gamma \sim 2$, and hydrogen column of $N_{H} \sim 1.9 \times 10^{23} \mathrm{H} \mathrm{cm}^{-2}$.

The large absorption may suggest that $1 \mathrm{E} 1743.1-2843$ is a background object behind our galaxy, such as an extragalactic AGN. No significant flux above the $35 \mathrm{keV}$ band with past observations, which indicates spectral turn-off near the energy $10-: 0 \mathrm{keV}$, may rule out the AGN possibility. We hence prefer galactic origin. From the spectral shape, a low mass X-ray binary (LMXB) near the Galactic Center is the most probable candidate. $\mathrm{X}$-ray luminosity is estimated to be $2 \times 10^{36} \mathrm{erg} \mathrm{sec}^{-1}$, a luminosity in which type-I bursts are expected. However, no burst has been detected through long term observations not only with $A S C A$ but also with other previous instruments, with perhaps more than $1200 \mathrm{ksec}$ exposure in total.

\section{References}

Bally, J. \& Leventhal, M. (1991), Nature, 353, 234

Bouchet, L. et al. (1991), ApJL, 383, L45

Koyama, K. et al. (1992), PASJ, 48, 249
Mirabel, I.F. et al. (1992), Nature, 358, 215

Pavlinsky, M.N. et al. (1994), ApJ, 425, 110

Sakano, M. et al. (1997), Proc of IAU Symp. 184 , ed. Y.Sofue 